\title{
Rapid Material Characterization of Deep-Alloyed Steels by Shock Wave-Based Indentation Technique and Deep Rolling
}

\author{
Tobias Czotscher $^{1} \mathbb{D} \cdot$ Nicole Wielki $^{2,3} \cdot$ Konstantin Vetter $^{1} \cdot$ Frank Vollertsen $^{1,3} \cdot$ Daniel Meyer $^{2,3}$
}

Received: 20 November 2018 / Revised: 9 January 2019 / Accepted: 11 January 2019 / Published online: 5 February 2019

(c) The Author(s) 2019

\begin{abstract}
Processing technologies such as laser deep alloying could enable the development of new construction materials based on micro-samples because of the rapid as well as flexible mixture of a base material with pre-deposited alloying elements. To maintain high-throughput material development, these new material compositions need to be characterized rapidly as well. High-throughput experimentation in material development is recognized as a novel and beneficial scientific way to generate knowledge and to identify new alloy compositions. This paper presents and compares different approaches for highthroughput characterization of different micro-samples produced by laser deep alloying. These approaches are based on plastic deformation by the mechanical indentation and reveal information about the material properties by rapidly measurable values called descriptors. The experiments indicate that the shock wave-based indentation technique and the deep rolling process are suitable methods to gain insights regarding the material properties of laser deep-alloyed micro-samples. Both processes show that the plastic deformation slightly increases with elevated laser power in laser deep-alloying process. Subsequent conventional measurements indicated that at higher laser power, lower hardness and lower amount of chromium result from the deep-alloying process. Moreover, local deviations of metallographic constituent, which results in larger hardness deviations, were predicted based on the descriptors.
\end{abstract}

Keywords Measurement · Deformation · Manufacturing process $\cdot$ High-throughput

\section{Introduction}

Conventional material developments are based on expensive experimental investigations of different material properties. Processing technologies such as laser deep alloying could enable the development of new construction materials because of the rapid as well as flexible mixture of a base material with pre-deposited alloying elements [1]. To maintain high-throughput material development, these new material compositions need to be characterized rapidly as well. The objective of realizing a specific performance profile of a material in a short time pursues the demand of efficient

Tobias Czotscher

czotscher@bias.de

1 BIAS - Bremer Institut für angewandte Strahltechnik GmbH, Klagenfurter Straße 5, 28359 Bremen, Germany

2 Leibniz-Institut für Werkstofforientierte Technologien - IWT, Badgasteiner Straße 3, 28359 Bremen, Germany

3 Universität Bremen and MAPEX, Bibliothekstraße 1, 28359 Bremen, Germany identification of new compositions. Thus, high-throughput experimentation in materials science has been recognized as a new approach to generate novel and beneficial scientific knowledge [2]. New high-throughput techniques must operate at least at the same accuracy level as comparable methods. The new techniques do not measure specific material properties directly [3]. Instead, values (so-called descriptive values) are linked to the specific material properties to measure more rapidly. Moreover, receiving information on material properties for micro-samples is not always possible with conventional methods, e.g., tensile tests. One example for deducing information from adapted processes could be a new method of hardness measurement. The hardness offers a descriptive value for the resistance of a material against the indentation of another material. However, the conventional indentation and evaluation process usually takes several seconds. A new method was presented in [4] and is based on laser-induced shock waves. The laser-induced shock wave indentation hardness measurement is further referred to as LiSE-hardness measurement. When energy density of a $\mathrm{CO}_{2}$ 
laser pulse exceeds a threshold, a plasma is induced, which results in a shock wave [5].

Laser-induced shock wave-based processes are commonly used such as for surface hardening of turbines to improve fatigue performance [6] or forming and joining operations [7]. Laser systems offer not only a high processing speed due to the fast expansion of the shock wave but also a highthroughput because laser scanners are highly dynamic.

The shock wave can also be used to push an indenter inside a material surface. Another well-known process is deep rolling, which causes plastic deformation of surface and sub-surface layers leading to an increase in compressive residual stress as well as a smoothing of the surface [8]. As the resistance of highly stressed components is important, the plastic deformation induced by deep rolling is conventionally used to optimize component properties [9]. Investigating the plastic deformation induced by deep rolling may reveal information about the material properties [10]. This paper presents and compares two different approaches for high-throughput characterization of different micro-samples produced by laser deep alloying. These approaches are based on plastic deformation by the mechanical indentation and give information about the material properties by rapidly measurable descriptors. The deformations introduced by these processes are characterized and analyzed to allow conclusions to be drawn about the material properties. The microstructure of the samples is varied by different ratios of base material to master alloy because of the variation of laser power in laser deep alloying. The goal of this paper is to understand the connection between the resulting plastic deformation and the subsequently assessed mechanical properties of the material as well as the microstructure in terms of the metallographic constituent.

\section{Processing of Laser Deep-Alloyed Materials}

Laser deep alloying describes the process of re-melting and mixing of the pre-deposited element layer with a base material. Figure 1 illustrates the experimental setup of the laser deep-alloying process. This method enables high-throughput material development of new alloy compositions in a small scale. The application of the master alloy layers was carried out by a laser beam melting (LBM) using a Realizer 250 machine with a $200-\mathrm{W}$ single-mode fiber laser and a wavelength of $1070 \mathrm{~nm}$.

The master alloy was applied with a layer thickness of $0.5 \mathrm{~mm}$ on a base plate. The master alloy layers were square shaped with a side length of $10 \mathrm{~mm}$. The dimensions of the base plate were $150 \times 150 \times 18 \mathrm{~mm}^{3}$, and 25 micro-samples were produced. Laser deep alloying was performed using a disk laser with a wavelength of $1030 \mathrm{~nm}$ and a maximum

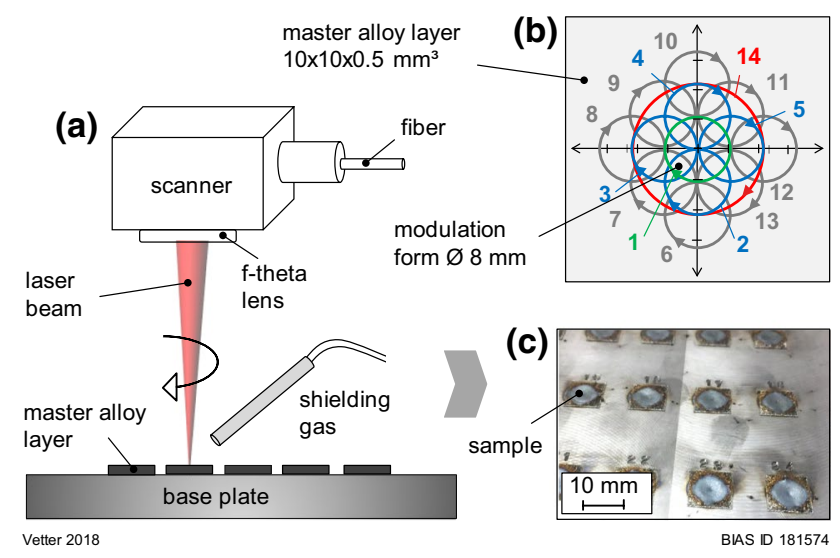

Fig. 1 a Principle of the laser deep alloying, $\mathbf{b}$ applied laser beam modulation form, $\mathbf{c}$ laser deep-alloyed samples after re-melting and mixing the pre-deposited master alloy layer with the base material

power of $12 \mathrm{~kW}$. The focusing optics was a 3D scanner optic with an f-theta lens with a focal length of $450 \mathrm{~mm}$ (cf. Fig. 1a). The spot diameter was $650 \mu \mathrm{m}$. Argon shielding gas was provided at a rate of 6 standard liters per minute laterally at an angle of $45^{\circ}$ using a nozzle with a diameter of $13 \mathrm{~mm}$. The nozzle was fixed at $20 \mathrm{~mm}$ from the process zone. The laser beam was modulated circularly by the scanner optics applying a geometrical form of 14 consecutive overlaying circles of two different sizes (as shown in Fig. 1b). Preliminary tests indicated that these modulations are suitable to achieve a homogeneous dilution of alloy elements [1]. The processing order of each circle was clockwise. The small circles were $2.44 \mathrm{~mm}$ in diameter. The large circle was $4.88 \mathrm{~mm}$ in diameter. The total modulation path length was $115 \mathrm{~mm}$. Applying this modulation form causes samples with a circular, symmetric shape with a diameter of $8 \mathrm{~mm}$ (Fig. 1c). For all experiments, the modulation form was applied at a modulation speed of $10 \mathrm{~m} / \mathrm{min}$. The laser power was varied from 3 to $5 \mathrm{~kW}$ to generate different melt volumes and chemical compositions. This range extends from the minimum laser power required to achieve a vapor capillary, which allows deep alloying to a maximal energy input that results in a microstructure with as little imperfection as possible [11]. The case hardening steel $\mathrm{C} 15$ with an initial hardness of $146 \mathrm{HV} 0.1 \pm 14 \mathrm{HV} 0.1$ was used as the base material. Atomized stainless steel X2CrNiMo17-12-2 was used for the generation of the master alloy layers on the base plate to produce the deep-alloyed samples. The selected material pairing allows a defined gradual change of the hardness in the alloy due to the varied chromium content at different melt volumes. The initial chemical compositions are listed in Table 1 for $\mathrm{C} 15$ and for the stainless steel X2CrNiMo17-12-2. The powder particles of the master alloy have a spherical morphology and a median particle size between $10 \mu \mathrm{m}$ and $45 \mu \mathrm{m}$. 
Table 1 Chemical composition of the base material $\mathrm{C} 15$ and the master alloy X2CrNiMo17-12-2 in weight percent (wt\%)

\begin{tabular}{llllccl}
\hline Material & $\mathrm{C}$ & $\mathrm{Si}$ & $\mathrm{Mn}$ & $\mathrm{Ni}$ & $\mathrm{Cr}$ & $\mathrm{Mo}$ \\
\hline C15 & 0.16 & 0.22 & 0.45 & 0.05 & 0.03 & 0.003 \\
X2CrNiMo17-12-2 & 0.012 & 0.56 & 0.31 & 13.1 & 16.5 & 2.27 \\
\hline
\end{tabular}

\section{Testing Methods}

\subsection{Metallographic Analysis}

The measured values for sample geometry, hardness and chemical composition were determined by means of metallographic cross-sections taken in the center of the samples. The arithmetic average of five samples was determined for the specification of the cross-sectional melt pool area. The analysis of the melt pool area is intended to draw conclusions about the melt volume because the samples are rotationally symmetric. Kalling etchant was applied on the cross sections for the analysis of the martensitic structures. Samples for Vickers micro-hardness testing were prepared by grinding and polishing the cross section. The hardness of the generated alloys was measured using a micro-hardness tester. An indentation load of $0.98 \mathrm{~N}$ was applied at a dwelling time of $15 \mathrm{~s}$. The distance between two hardness impressions was $0.2 \mathrm{~mm}$. The evaluation was carried out at $50 \times$ magnification. The measurements were taken over the cross-sectional area at $0.2 \mathrm{~mm}$ from the surface and for comparison also directly on the surface. The number of indentations was between 34 and 40, depending on the cross-sectional size of the sample. The arithmetic average of the hardness impressions from three samples with standard deviation was taken. The chemical analysis was made using EDX with the scanning electron microscope. The measurements were taken over the cross-sectional area at $0.2 \mathrm{~mm}$ from the surface. The distance between two measurement points was $0.6 \mathrm{~mm}$. The arithmetic average with standard deviation was taken.

\subsection{LiSE-Hardness Measurement}

The LiSE-hardness measurement experiments were conducted with a nanosecond pulsed TEA $\mathrm{CO}_{2}$ laser. The schematic setup is shown in Fig. 2. The pulse duration of the laser beam is $100 \mathrm{~ns}$ with a maximal pulse energy of $6 \mathrm{~J}$. Accordingly, an intensity of $1.5 \mathrm{GW} / \mathrm{cm}^{2}$ is reached with a minimal quadratic beam spot of $4 \mathrm{~mm}^{2}$ at a focal length of $200 \mathrm{~mm}$. The spatial energy distribution of the laser beam is a top hat with a near-uniform fluence. The high-intensity laser beam creates a plasma on top of the indenter, which becomes instable and results in a shock wave of several MPa [12]. The expanding shock wave pushes the indentation ball within microseconds inside the test sample. The laser pulse energy was set to a maximum of $6 \mathrm{~J}$ during the

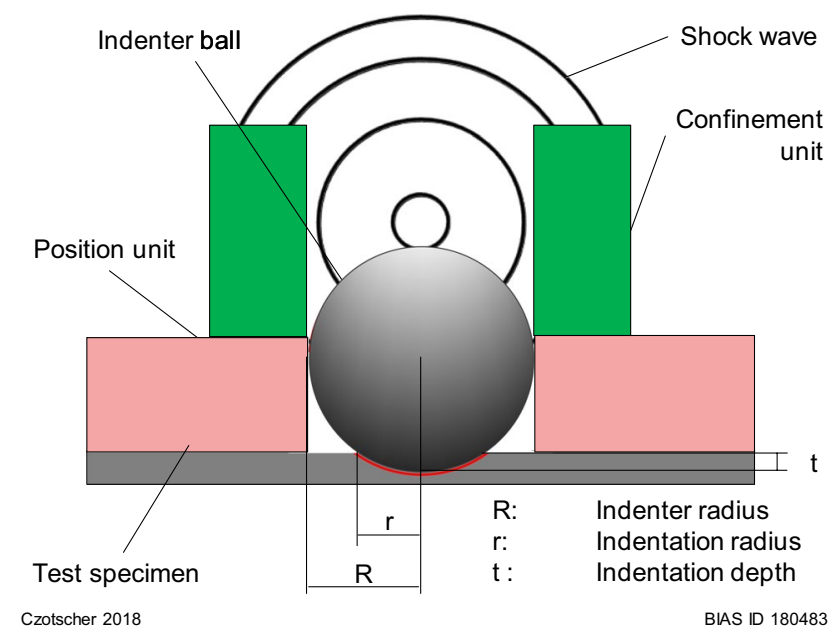

Fig. 2 Schematic view of indentation test with confinement unit

experiments. Indentations were created on the laser deepalloyed material with $\mathrm{Al}_{2} \mathrm{O}_{3}$ indenter balls. The shock wave energy transfer efficiency and impact efficiency strongly depend on the indenter diameter [13]. Smaller indenter diameters are recommendable for higher efficiencies. Thus, the indenter balls had a diameter of $4 \mathrm{~mm}$. A pressure reflection unit was placed on top of the position unit to increase the acting shock wave pressure on the indenter ball. The created indentations were analyzed according to standard hardness test method for metallic materials (EN ISO 6506). The evaluation was carried out at $20 \times$ magnification with the laser microscope VK9710 from Keyence. Additional to the indentation diameter, the depth of the indentation was measured as indicated in Fig. 2.

\subsection{Deep Rolling}

Deep rolling experiments were carried out to investigate the material properties of the laser deep-alloyed specimens. As deep rolling induces a mechanical impact by forming the workpiece material without material removal, the workpiece is plastically deformed due to the contact with the pressurized tool. Depending on the process parameters, the material properties and the microstructure, the translational movement of the tool results in tactilely detectable tracks on the workpiece (cf. Fig. 3) [14]. The descriptors track width $b_{t}$ and track depth $z_{t}$ were measured after the deep rolling process with a roughness measurement device, which has a point angle of $90^{\circ}$. The tactile measurements were taken 


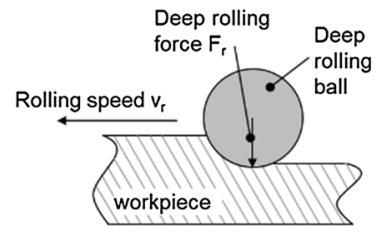

Sectional side view

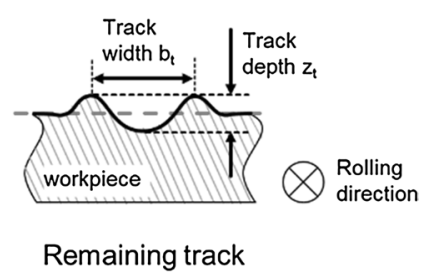

Fig. 3 Scheme of the plastic deformation induced by a deep rolling process

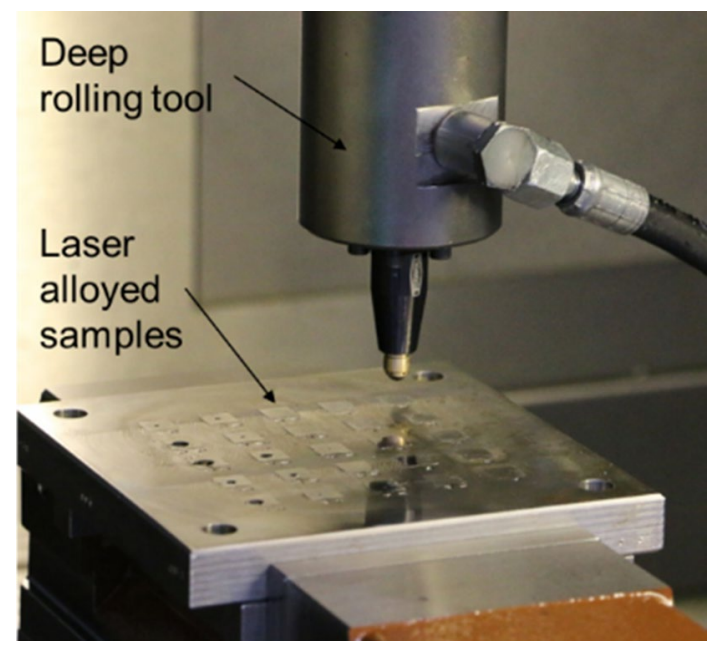

Fig. 4 Scheme of the plastic deformation induced by a deep rolling process

at intervals of about $1 \mathrm{~mm}$ along the track of the sample to detect changes in hardness and material composition. Moreover, the determined track depths and widths allow a description of the influence of different laser powers. Since previous investigations have shown that the descriptors react more sensitively to changes in the material when lower deep rolling pressures are used [10], a deep rolling pressure of 85 bar was chosen for the investigation. 85 bar results in average in a fixed rolling force of $217 \mathrm{~N}$. A hydrostatic tool with a ball diameter of $6 \mathrm{~mm}$ from Ecoroll (HG6) was used in combination with a $5 \%$ emulsion as pressure medium. Deep rolling was carried out at a feed speed of $100 \mathrm{~m} / \mathrm{min}$. Figure 4 shows the experimental setup. The deep rolling force Fr, which represents another descriptor, was measured using a Kistler dynamometer.

\section{Results}

\subsection{Analysis of Laser Deep-Alloyed Samples}

The cross-sectional areas of the samples produced with different laser powers are obtained in Fig. 5. They illustrate the

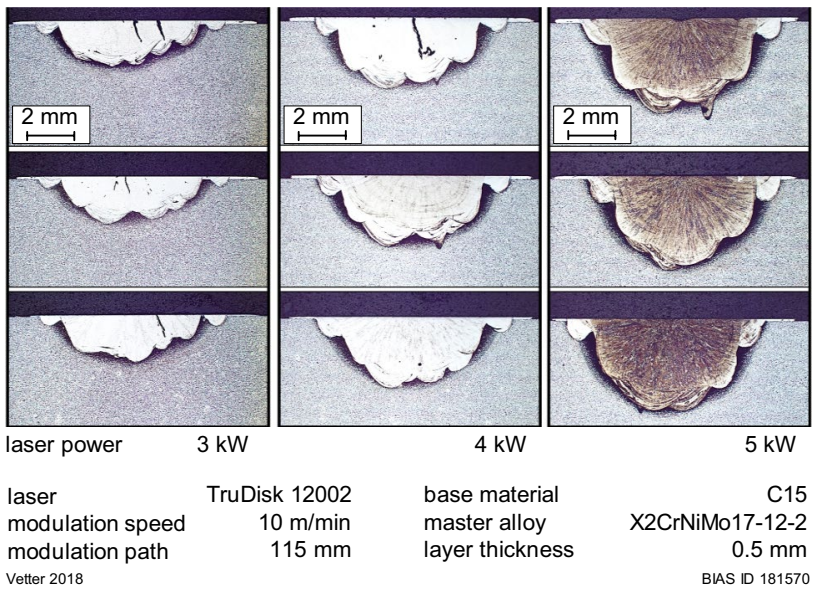

Fig. 5 Cross sections of three samples each, produced with different laser powers of $3 \mathrm{~kW}, 4 \mathrm{~kW}$ and $5 \mathrm{~kW}$
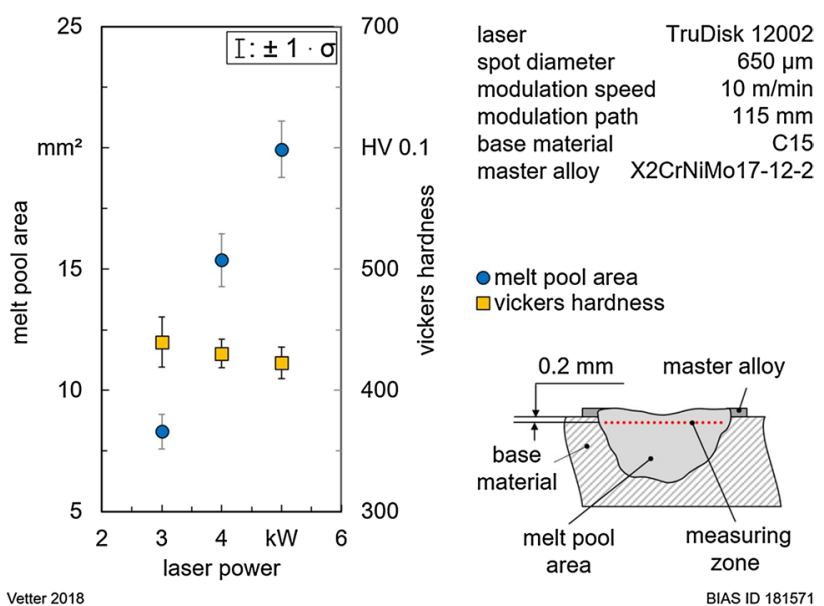

Fig. 6 Melt pool area and Vickers hardness depending on the laser power

increase in melt volume with increasing laser power. The samples are mainly free of pores. Cracks were found underneath the surface for laser powers of $3 \mathrm{~kW}$ and sporadically for laser powers of $4 \mathrm{~kW}$. For higher laser powers of $5 \mathrm{~kW}$, no cracks occurred, but some asperities were observed in the center regions of the former melt pool. With increasing laser power from 3 to $5 \mathrm{~kW}$, the melt pool area increases from 8.3 to $19.9 \mathrm{~mm}^{2}$ (Fig. 6). Between 3 and $4 \mathrm{~kW}$, the increase in melt pool area is $85 \%$. With a further increase in laser power to $5 \mathrm{~kW}$, the melt pool area increases further by $29 \%$. The diagram shows that the hardness of the samples is affected by the increasing melt pool area. The micro-hardness decreases from 440 HV0.1 to 423 HV0.1. The deviation of the measured values is lowest for samples generated with a laser power of $4 \mathrm{~kW}$, whereas it is highest for samples generated with $3 \mathrm{~kW}$. 

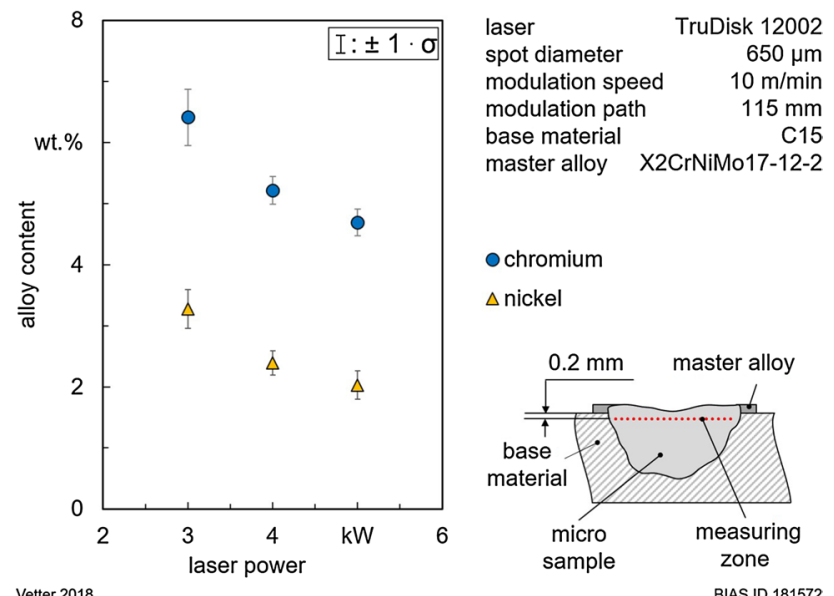

Fig. 7 Average chromium and nickel content close to the surface depending on the laser power

Figure 7 shows the average chromium and nickel content over the cross-sectional area of the sample as a function of the laser power. The measurement was taken close to the surface at a depth of $0.2 \mathrm{~mm}$. Both the chromium content and the nickel content decrease with increasing melt volume, indicated by the melt pool area because of the increasing laser power. The standard deviation of the values shows only a small spread. Between $3 \mathrm{~kW}$ and $4 \mathrm{~kW}$, the decrease in chromium content is $19 \%$. With a further increase in laser power to $5 \mathrm{~kW}$, the chromium content decreases further by $10 \%$.

Figure 8 shows the deviation of hardness and chromium content within a sample generated with a laser power of $4 \mathrm{~kW}$. The chromium content was measured at $0.2 \mathrm{~mm}$ underneath the surface. The micro-hardness was measured both, below and directly on the surface. The chromium

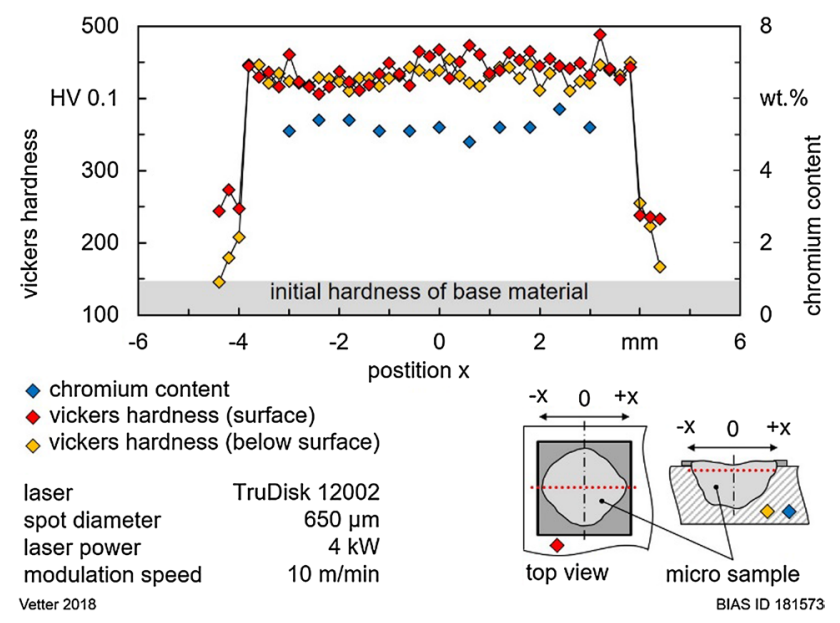

Fig. 8 Micro-hardness and chromium content within a sample generated with a laser power of $4 \mathrm{~kW}$ and a comparison of micro-hardness measured $0.2 \mathrm{~mm}$ below and directly on the surface content is between 4.8 and $5.7 \mathrm{wt} \%$. On the surface, the average micro-hardness of $441 \mathrm{HV} 0.1$ is slightly higher than below the surface with an average of 431 HV0.1. However, higher deviations are observed in micro-hardness at the surface in a range between $406 \mathrm{HVO} 0.1$ and $488 \mathrm{HV} 0.1 \mathrm{com}-$ pared to those below the surface. Below the surface, the hardness varies between $410 \mathrm{HVO} 0.1$ and $454 \mathrm{HV} 0.1$.

Figure 9 reveals the differences in the microstructure. The base material $\mathrm{C} 15$ consists of different grain sizes. Ferrite and perlite are found in the base material. The deep-alloyed material is etched with Kalling etchant, and depending on the used laser power, the martensitic structure is formed differently. $3 \mathrm{~kW}$ shows a rather inhomogeneous microstructure compared to $4 \mathrm{~kW}$ and $5 \mathrm{~kW}$ laser powers.

\subsection{Analysis of Deep Rolled Samples}

By analyzing the process forces in deep rolling, first information is gained during the processing. Changes in, for example, workpiece hardness or topography are visible in a slight increase or decrease in the rolling force $\mathrm{F}_{\mathrm{r}}$. The force curve is shown in Fig. 10 as an example for a laser deepalloyed sample produced with $3 \mathrm{~kW}$ laser power. Regarding the force curve, different areas along the track can be found.

The base material has the lowest hardness and the highest roughness due to the pre-milling of the surface. All measured forces show oscillating behavior in this area. In contrast, the alloyed area has a polished surface due to the sample preparation so that fluctuations in force cannot be traced back to the topography. The sudden increase in the feed force clearly shows the entering of the deep rolling tool into the laser deep-alloyed area. It can also be deduced from the sudden drop of this force where the tool has left the laser deepalloyed area again. In the example shown, the deep rolling force has a constant level between investigated positions 1-5. Only in the middle region of the laser deep-alloyed sample (position 3), a different behavior can be observed. Further
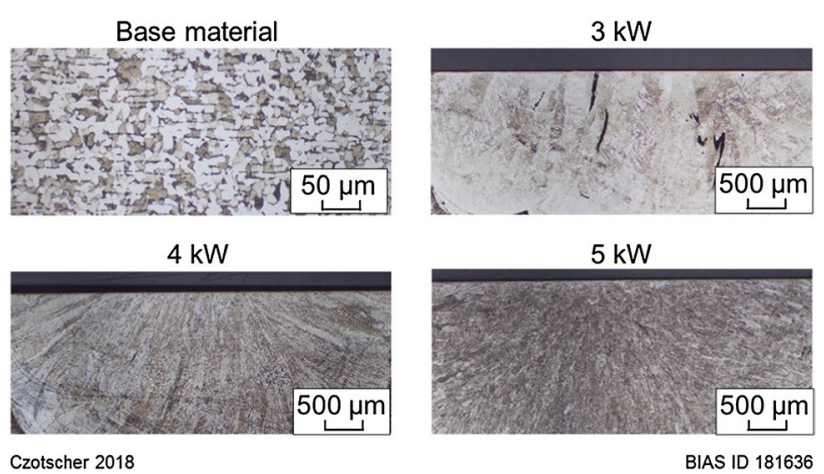

Fig. 9 Comparison of base material with martensitic microstructures produced by laser deep alloying with different laser powers $(3 \mathrm{~kW}$ to $5 \mathrm{~kW}$ ) and etched with Kalling etchant 


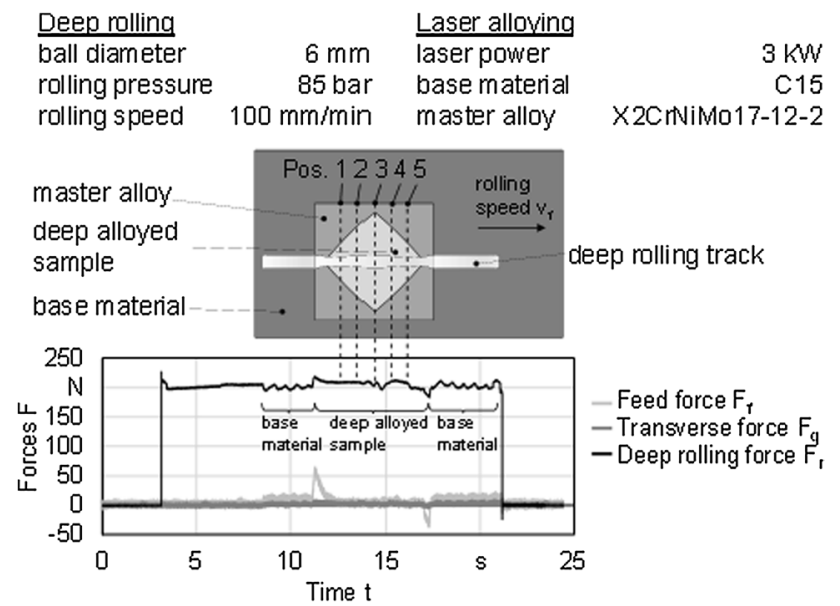

Fig. 10 Exemplary visualization of the measured force curves

information can be determined by investigating the plastic deformation induced by the deep rolling process. As track depth and width are influenced by the material flow behavior and material properties, e.g., the hardness and yield strength, changes within the area of a laser deep-alloyed sample could be observed for varied material composition. Several tactile measurements are taken at intervals of one millimeter along the track (cf. Fig. 10: position 1-5). The highest values are obtained in the middle of the samples, independent of the laser power (cf. Fig. 11). For a laser power of $5 \mathrm{~kW}$, a crater was found in the middle of the sample, which made it impossible to measure the track geometry. The gradient of values determined within a sample decreases with increasing laser power. The depths and widths are set to constant levels, and the standard deviation of the results decreases with increasing laser power.
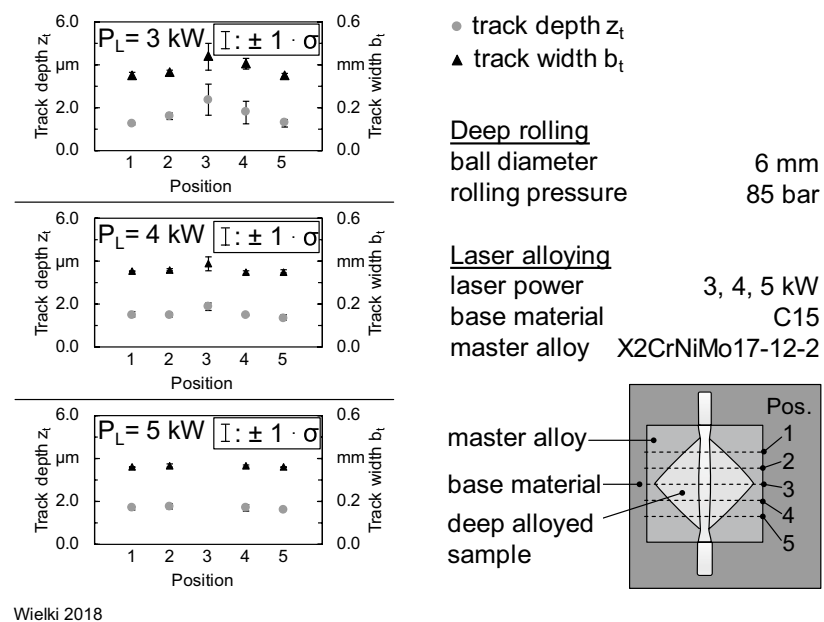

Wielki 2018

Fig. 11 Determined descriptors for different laser powers within the deep-alloying process
As the hardness was determined to decrease with increasing laser power, it was expected that both the track widths and the track depths in deep rolling would increase with lower material hardness. Instead, the track width increases slightly with higher material hardness. The different behavior of track depth and track width could be traced back to elastic behavior of the indenter. An elastic deformation of the tool ball during the deep rolling process could lead to the observed deviations.

\subsection{Analysis of LiSE-Hardness Indentations}

The measured laser-induced indentation geometries are shown in Fig. 12 for the deep-alloyed materials and the base material. The results demonstrate that the created indentation depth and indentation diameter increase when higher laser powers are used during laser deep alloying. The highest indentation depths and diameters are measured on the untreated base material. The calculated depth and diameter deviation are the smallest for the $4 \mathrm{~kW}$ laser power deep-alloyed samples. For lower laser powers of $3 \mathrm{~kW}$ and for higher laser powers of $5 \mathrm{~kW}$, the measured depth and diameter deviation increase. It can also be obtained that the standard deviations are high for the measured indentation diameter and depth. This is especially the case for the untreated samples. The minimum and maximum deviations are indicated by the horizontal broken lines for the measurements on the base material. Furthermore, the coefficient of variation is determined for the created diameter, the indentation depth and from the hardness measurements on the samples. The coefficient of variation describes the ratio of the standard deviation to its mean value. Larger measured values may lead to larger absolute deviations. This stochastic parameter describes the repeatability of a measurement and
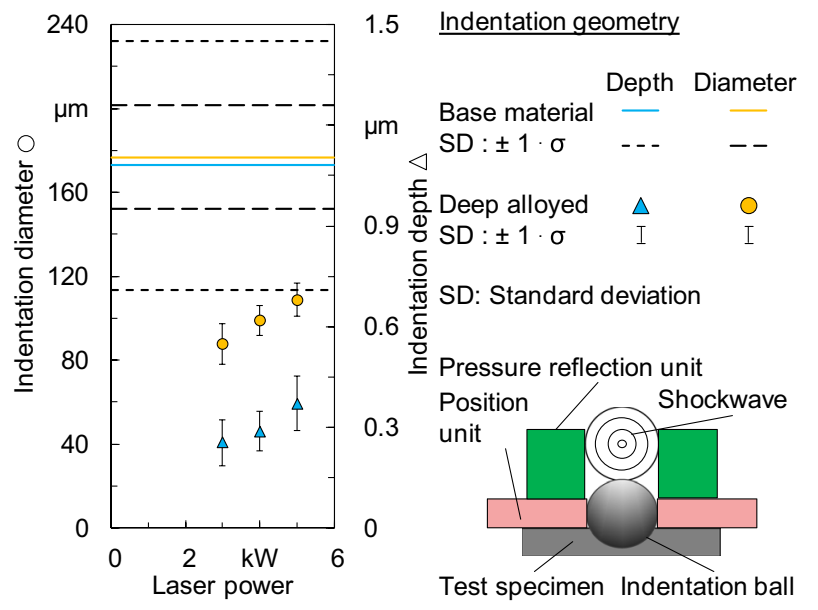

Czotscher 2018

BIAS ID 180217

Fig. 12 Indentation depth and indentation diameter created in laser deep-alloyed material 


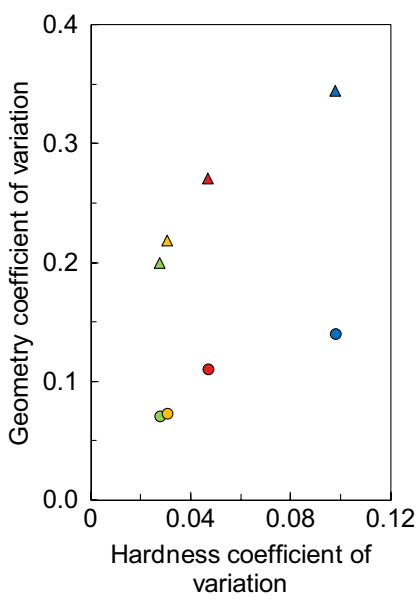

Czotscher 2018
Coefficient of variation

$\begin{array}{lcc}\text { Sample } & \text { Depth } & \text { Diameter } \\ \text { Base } & \Delta & 0 \\ 3 \mathrm{~kW} & \Delta & 0 \\ 4 \mathrm{~kW} & \triangle & 0 \\ 5 \mathrm{~kW} & \triangle & 0\end{array}$

Pressure reflection unit

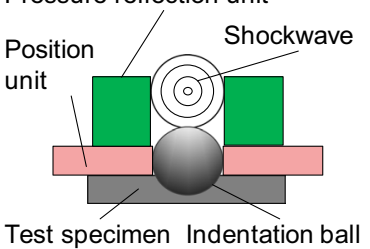

BIAS ID 180217

Fig. 13 Comparison between coefficient of variation determined by conventional hardness measurements and by LiSE-hardness measurement for base material and the laser deep-alloyed materials

makes it comparable to other measurement results. Thus, the coefficient of variation for the indentation diameter and the indentation depth is compared to the hardness coefficient of variation determined by conventional Vickers hardness measurements.

It is shown in Fig. 13 that a larger coefficient of variation also leads to a larger coefficient of variation for the indentation depth and indentation diameter. The largest coefficients of variation are calculated for the base material, whereas the second largest coefficients of variation are obtained for the $3 \mathrm{~kW}$ laser deep-alloyed sample. The lowest coefficients of variation are observed for the $4 \mathrm{~kW}$ sample. It can also be observed that the coefficient of variation for the diameter is on the average 2 times higher than the hardness coefficient of variation. The coefficient of variation for the indentation depth is on the average 6.5 times higher compared to the hardness coefficient of variation.

\subsection{Comparison Between Methods}

The descriptors determined utilizing the two methods presented above are plotted as plastic deformation over the average Vickers hardness of the laser deep-alloyed samples in Fig. 14. To compare the two methods, average values of the descriptors for each method are shown in this graphic. The position dependency is not considered, since it cannot be guaranteed that the values were determined at comparable positions using both methods. The comparison between the measurements shows that the indentation depth, the indentation diameter and track depth correlate with the hardness. A larger hardness value results in a lower geometrical value. This behavior cannot be clearly confirmed for the track

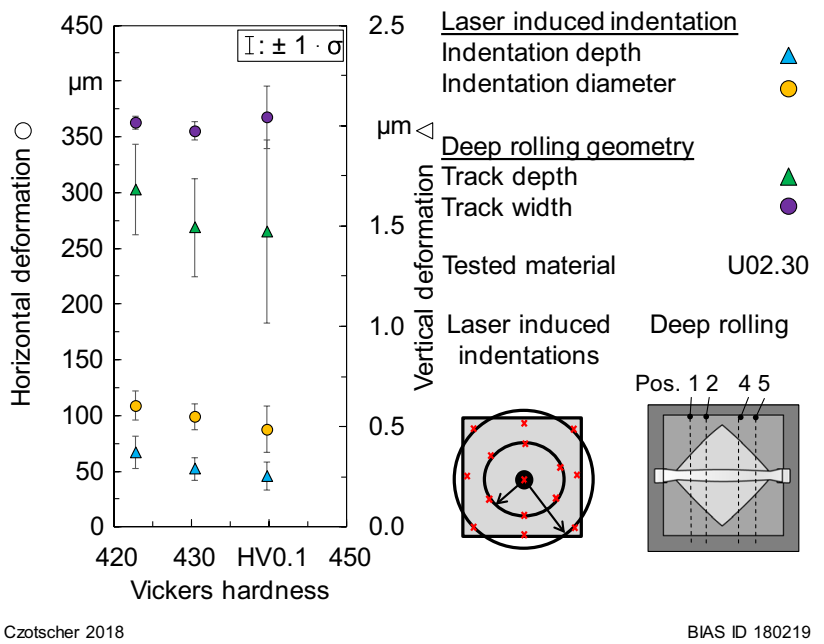

Fig. 14 Comparison of induced plastic deformation sizes between the different measurement methods in laser deep-alloyed materials

width, since the value determined at the highest hardness differs from the mentioned trend.

\section{Discussion}

The laser power influences the mixing ratio of pre-deposited master alloy and soft base material during laser deep alloying. Figure 9 shows the different metallurgical cross sections. In the unprocessed base material, characteristic ferrite and perlite phases as well as different grain sizes are obtained. Instead, only martensitic structures are found in the laser deep-alloyed materials processed with different laser powers. Small and fine-dispersed grains are observed in these martensitic structures. By comparing the base- and laser-processed material, it is obvious that the base material results in higher coefficients of variation in Vickers hardness. Moreover, the $3 \mathrm{~kW}$ samples reveal a rather inhomogeneous martensitic structure compared to the other laser deep-alloyed samples. No visible differences are obtained between the $4 \mathrm{~kW}$ and $5 \mathrm{~kW}$ laser deep-alloyed martensitic phases. A further explanation offers Fig. 7. The laser power influences the mixing ratio of pre-deposited master alloy and soft base material during laser deep alloying. Larger deviations and amounts of chromium are found in the $3 \mathrm{~kW}$ processed material. In contrast, higher laser powers lead to lower amounts of chromium and nickel in laser deepalloying processes. It can be derived from Figs. 6 and 7 that the melt area and, thus, the melt volume are congruent to the measured alloy content. Thus, the lower material hardness could be a result of a larger melt volume and, accordingly, of a lower amount of chromium in the alloyed sample. Larger deviations and amounts of chromium could lead to the observed Vickers hardness deviations because chromium 
is a strong carbide-forming element, which increases the hardness of a material. Like the conventional hardness deviations, the LiSE-hardness measurement process shows that it is sensitive to changes in metallographic constituent and local deviations as indicated in Fig. 13. These deviations observed by the conventional and LiSE-hardness measurement were not detected by deep rolling. This could be explained by higher pressures of 85 bar, which are applied during deep rolling. These pressures result in an average load of $217 \mathrm{~N}$. The deep rolling process is more effected by imperfections and cracks underneath the surface compared to the hardness measurement where $0.98 \mathrm{~N}$ was applied. The highest descriptor deviations of the laser deep-alloyed samples were also observed during deep rolling on the $3 \mathrm{~kW}$ samples. Particularly, the deep rolling process with 85 bar shows high track deviations in the center region. Cracks are mainly found underneath the surface in the center region as shown in Fig. 5. Higher laser powers such as $5 \mathrm{~kW}$ and even $6 \mathrm{~kW}$ could cause a recoil pressure during the laser processing and, accordingly, to an ejection of molten material. This may explain the asperity in the center region of the melt pool area for higher laser powers. In this case, it was not possible to evaluate the deep rolled track (as shown in Fig. 11). Thus, samples produced with $3 \mathrm{~kW}$ and with $5 \mathrm{~kW}$ laser powers reveal higher Vickers hardness deviations. Instead, samples produced with $4 \mathrm{~kW}$ laser power offer the best results in terms of lowest hardness deviations. These findings could also be observed by LiSE-hardness measurement. Additionally, the results of the LiSE-hardness measurements revealed that the indentation diameter results in a lower coefficient of variation values compared to the depth values.

Thus, the experiments reveal that LiSE-hardness measurement and deep rolling process are suitable methods to characterize the material properties of laser deep-alloyed micro-samples even with minor gradation in metallurgical constituent. Both processes show that the plastic deformation slightly increases with elevated laser power in the laser deep-alloying process (Fig. 14). Local deviations in metallographic constituent, which results in larger hardness deviations, can also be obtained by LiSE-hardness measurement. Both processes complement one another because the LiSEhardness measurement is sensitive to local changes in metallurgical constituent and deep rolling is more sensitive to imperfections and cracks underneath the material surface.

\section{Conclusions}

A laser deep-alloying micro-manufacturing process and two novel measurement methods, namely deep rolling and laserinduced indentations, were analyzed and presented in this paper. The following conclusions can be drawn:
- Even small gradation in metallurgical constituent, which result in small changes in hardness and imperfections underneath the surface, could be detected by both measurement processes, which makes them suitable as descriptors for further analysis and high-throughput material characterization.

- The conducted LiSE-hardness measurements reveal that the diameter values are preferred over depth values to predict the material hardness and local deviations in metallographic constituent. For further experiments, the coefficient of variation of the diameter can be used.

- The LiSE-hardness measurement and the deep rolling process are suitable high-throughput methods to identify correlations and to gain new insights regarding the material properties of metallic alloy compositions.

Acknowledgements Financial support of the subprojects D02 "Laser induced hardness measurements," U02 "Laser deep alloying for the realization of finely graded alloys" and U04 "Mechanical Treatment" funded by the Deutsche Forschungsgemeinschaft (DFG, German Research Foundation) - Project Number 276397488 - SFB 1232 is gratefully acknowledged.

Open Access This article is distributed under the terms of the Creative Commons Attribution 4.0 International License (http://creativeco mmons.org/licenses/by/4.0/), which permits unrestricted use, distribution, and reproduction in any medium, provided you give appropriate credit to the original author(s) and the source, provide a link to the Creative Commons license, and indicate if changes were made.

\section{References}

1. Vetter K, Freiße H, Vollertsen F (2017) High-throughput material development using selective laser melting and high power laser. In: Proceedings of the 7th WGP-Jahreskongress, Aachen, Germany, pp 511-518

2. Potyrailo R, Rajan K, Stoewe K, Takeuchi I, Chisholm B, Lam H (2011) Combinatorial and high-throughput screening of materials libraries: review of state of the art. ACS Comb Sci 13(6):579-633

3. Mädler L (2014) Is high-throughput screening for structural mate$\mathrm{rial} / \mathrm{metals}$ possible?, 4th NanoMan, Bremen, Germany

4. Czotscher T (2018) Analysis of TEA-CO - -laser-induced plasma to establish a new measurement technique. J Laser Appl 30(3):032604-1-032604-5

5. O'Keefe JD, Skeen CH, York CM (1973) Laser-induced deformation modes in thin metal targets. J Appl Phys 44(10):4622-4626

6. Montross CS, Wei T, Ye L, Clark G, Mai Y (2002) Laser shock processing and its effects on microstructure and properties of metal alloys: a review. Int J Fatigue 24(10):1021-1036

7. Veenaas S, Vollertsen F (2014) High speed joining by laser shock forming. Adv Mater Res 966-967:597-606

8. Gariépy A, Miao HY, Lévesque C (2017) Simulation of the shot peening process with variable shot diameters and impacting velocities. Adv Eng Softw 114:121-133

9. Schulze V (2006) Modern mechanical surface treatment: States, stability, effects. Wiley-VCH Verlag, Weinheim

10. Kämmler J, Guba N, Vetter K, Vollertsen F, Meyer D (2018) Material characterization by deep rolling of laser deep alloyed 
micro-samples. In: Proceedings of the euspen. International Conference and Exhibition, Venice, Italy, pp 335-336

11. Vetter K, Hohenäcker S, Freiße H, Vollertsen F (2017) Use of additive manufacturing for high-throughput material development. In: Laser Manufacturing Conference (LiM), Munich, Germany

12. Vollertsen F, Niehoff HS, Wielage H (2009) On the acting pressure in laser deep drawing. Prod Eng 3:1-8
13. Czotscher T (2018) Material characterisation with new indentation technique based on laser-induced shockwaves. Lasers Manuf Mater Process 5(4):439-457

14. Kämmler J, Wielki N, Meyer D (2018) Surface integrity after internal load oriented multistage contact deep rolling. Procedia CIRP 71:490-495 